\title{
FORMULATION AND EVALUATION OF LEVOFLOXACIN-CHITOSAN / $\beta$ - CYCLODEXTRIN NANOPARTICLES BY IONIC GELATION
}

\author{
Baby Beny*1, Swamy Vrushabendra BM ${ }^{2}$, Jayaveera $\mathrm{KN}^{3}$, Abraham Abin ${ }^{4}$ Prasanth VV \\ ${ }^{1}$ Department of Pharmaceutics, Karnataka College of Pharmacy, Bangalore-560064, Karnataka, India \\ ${ }^{2}$ Department of Pharmacology, East Point College of Pharmacy, Bangalore-560038, Karnataka, India \\ ${ }^{3}$ Department of Chemistry, Jawaharlal Nehru Technological University, Anantapur-560005, Andhra Pradesh, India \\ ${ }^{4}$ Department of Pharmaceutics, Gautham College of Pharmacy, Bangalore-560032, Karnataka, India
}

Received 10 Feb 2015; Review Completed 05 March 2015; Accepted 08 March 2015, Available online 15 March 2015

\begin{abstract}
Background: Levofloxacin is a broad spectrum anti-infective agent, which is rapidly and completely absorbed after oral administration. The half life of Levofloxacin is $6-8 \mathrm{~h}$ after conventional dosing. The objective of the present work was to develop Levofloxacin nanoparticles to retain the dosage form in the absorption site more than the half life of the drug, enhance the bioavailability of drugs, reduce dose frequency, toxicity and patient compliance.

Methods: The compositions of different formulations of Levofloxacin nanoparticles by the ionic gelation method using biodegradable polymer chitosan and tripolyphosphate as cross linking agent.

Result and Discussion: The particle size lies of the prepared nanoparticles between 199 and $369 \mathrm{~nm}$ and the drug content found between $51.13 \pm 0.28$ and $71.12 \pm 0.14 \%$. The particle size of nanoparticles increased with increasing concentration of polymer matrix density and this may be due to the increased viscosity of the inner phase and decreased with increasing concentration of $\beta$-cyclodextrin. Scanning electron microscopy indicated that the prepared nanoparticles were discrete, uniform and spherical with a smooth surface. The in vitro release showed that the drug release from the prepared nanoparticles was characterized by an initial fast release and followed by a delayed release phase. During and at the end of the accelerated stability study, the tested formulation showed almost same drug content, in vitro drug release and no colour changes were observed from that observed at the opening of the study.

Conclusion: Among all the formulations (GIA, GIB, GIC, GID, GIE and GIF), the formulations G1C, G1E and G1F followed the drug release in a controlled manner. The in vitro release profile showed that this is a potential drug delivery for Levofloxacin and has to confirm in the in vivo settings as a separate investigation in future.

Key words: Controlled drug delivery, In vitro drug release, Nanoparticle, Particle size, Stability studies, Surface morphology
\end{abstract}

\section{INTRODUCTION}

Levofloxacin is a broad spectrum anti-infective agent, which is rapidly and completely absorbed after oral administration. The half life of Levofloxacin is 6-8 h after conventional dosing ${ }^{1}$. Recently the research interest is evolving to develop a drug delivery system with the use of biodegradable polymers. Nanoparticles have huge awareness as potential drug delivery devices in the appliance of controlled and targeted release for various absorptive tissues by improving the intracellular penetration. Moreover it represents an attractive alternative for improving the modulation of drug delivery, shelf life and stability in biological fluids ${ }^{2}$.

Oral drug delivery is the most attractive and favoured manner of drug delivery for achieving mutually systemic and local therapeutic effects. But a variety of problems are also related with the conventional oral dosage forms, that it is frequently essential to take several times per day to retain the concentration of administered drug within the therapeutically effective range which results in a fluctuated drug level and consequently undesirable toxicity and poor efficiency. So to overcome such problems associated with conventional oral dosage form, the idea of controlled drug delivery systems was introduced $^{3}$.

The real challenge in the development of a controlled drug delivery system is not just to control the drug release, also to extend the existence of the dosage form in the absorption site until all the drug is completely released in the preferred period of time ${ }^{4-6}$.

*Address for Correspondence Beny Baby* Assistant Professor Department of Pharmaceutics Karnataka College of Pharmacy Thirumenahalli, Hegde Nagar main road Bangalore - 560 064, Karnataka, India E-mail:benykarakat@gmail.com 
Continuous release of the drug involves polymers that release the drug at a controlled manner due to the degradation of polymer over time and it can be achieved by using drug carrying polymer.

In the present work, our aim was to develop a new nanoparticulate drug carrier that combines the benefits of chitosan nanoparticles and cyclodextrins to enhance the bioavailability of drugs, retain the drug in the absorption site more than the half life of the drug, reduce dose frequency, toxicity and patient compliance.

\section{MATERIALS AND METHODS}

\subsection{Materials}

Levofloxacin was a gifted sample from Orchid Chemicals, Chennai, India. Chitosan (low molecular weight) and Sodium TriPolyphosphate were purchased from Central Fisheries Department, Cochin, India. Sodium hydroxide and acetic acid was supplied from Merck (India) Ltd, Mumbai. The $\beta$-cyclodextrin was provided from Yarrow Chemicals, Mumbai, India. All other reagents used were of analytical grade.

\subsection{Methods}

\subsubsection{Solubility studies}

Higuchi and Connors method were used to determine the phase solubility ${ }^{7}$. Levofloxacin which exceeded its solubility in phosphate buffer solution ( $\mathrm{pH}$ 6.8), was transferred to a screw capped vials containing $25 \mathrm{~mL}$ of aqueous solution of $\beta$-cyclodextrin at various concentrations $(0,5,7.5,10,13$ and $15 \mathrm{mM}){ }^{[8]}$. The above mixture was stirred for $48 \mathrm{~h}$, at $37 \pm 0.1{ }^{\circ} \mathrm{C}$ and $2000 \mathrm{rpm}$ on a rotary shaker to obtain equilibrium of mixture. After reaching equilibrium, samples were filtered through membrane filter $(100 \mu \mathrm{m})$ and after suitable dilution, assayed spectrophotometrically (Shimadzu 1800, Japan) at $287 \mathrm{~nm}$.

\subsubsection{Formulation of Levofloxacin -chitosan/ $\beta$ - cyclodextrin nanoparticles}

Complexation of Levofloxacin with $\beta$-cyclodextrin was developed using liquid / liquid co-precipitation method 8 , 9 . The composition of different formulations of Levofloxacin nanoparticles by the ionic gelation method is shown in Table 1. Chitosan gel solution $(0.2 \% \mathrm{w} / \mathrm{v})$ was prepared using glacial acetic acid and stabilized overnight to obtain clear solution ${ }^{[10]}$. Tripolyphosphate solution $(0.1,0.3,0.5 \% \mathrm{w} / \mathrm{v})$ were prepared in distilled water and added drop wise with a syringe to the above gel solution with constant stirring. The prepared Levofloxacin - $\beta$-cyclodextrin complex (different ratios) was added to the above chitosan gel solution and the resulting suspension was subsequently centrifuged at $10000 \mathrm{rpm}$ for $10 \mathrm{~min}$, further the pellets obtained were resuspended in deionised water by sonication, then centrifuged and dried at room temperature $^{11,12}$.

Table 1: Compositions of LEV- chitosan / $\beta$-cyclodextrin nanoparticles

\begin{tabular}{|c|c|c|}
\hline Formulations & Levofloxacin: Chitosan: $\beta$-cyclodextrin & Tripoly Phosphate (\%) \\
\hline G1A & $1: 1: 1$ & 0.1 \\
\hline G1B & $1: 2: 1.5$ & 0.1 \\
\hline G1C & $1: 3: 2$ & 0.3 \\
\hline G1D & $1: 1: 2.5$ & 0.3 \\
\hline G1E & $1: 2: 3$ & 0.5 \\
\hline G1F & $1: 3: 3.5$ & 0.5 \\
\hline
\end{tabular}

\subsection{Evaluations}

\subsubsection{Physicochemical and Morphological characterization of Nanoparticles}

Particle size determinations (Malvern Particle Size Analyzer, Mastersizer 2000, USA), of samples were prepared by dispersing nanoparticles with sufficient amount of water to achieve obscuration around $5 \%$. From the particle size distribution data, the average particle size was determined ${ }^{12}$.

\subsubsection{Encapsulation efficiency of Levofloxacin}

For this, nanoparticles were dissolved in ethanol and ultracentrifuged at $10000 \mathrm{rpm}$ for $30 \mathrm{~min}$. The supernatant solution was separated and assayed at 287 $\mathrm{nm}$. The encapsulation efficiency of Levofloxacin was calculated as follows ${ }^{13,14}$.

$$
\text { Entrapment efficiency }(\%)=(\mathrm{Tp}-\mathrm{Tf}) 100 / \mathrm{Tp}
$$

Where,

$$
\begin{aligned}
& \mathrm{Tp}=\text { Total LEV used } \\
& \mathrm{Tf}=\text { Free LEV in the supernatant }
\end{aligned}
$$

\subsubsection{Drug content}

$100 \mathrm{mg}$ of Levofloxacin loaded nanoparticles were dissolved in phosphate buffer $(\mathrm{pH}$ 6.8) and stirred in sonicator for $4 \mathrm{~h}$ at $30{ }^{\circ} \mathrm{C}$. The samples were filtered using $0.2 \mu \mathrm{m}$ filter and assayed at $287 \mathrm{~nm}$.

\subsubsection{In vitro release study}

At first $900 \mathrm{~mL}$ of $0.1 \mathrm{~N} \mathrm{HCl}(\mathrm{pH} \mathrm{1.2)}$ was used as the dissolution medium for $2 \mathrm{~h}$ and then same volume of phosphate buffer ( $\mathrm{pH}$ 6.8) was replaced in the dissolution apparatus for $10 \mathrm{~h}$ at $50 \mathrm{rpm}$ and temperature was maintained at $37 \pm 1{ }^{\circ} \mathrm{C}$. USP XXIII, type-II dissolution test apparatus was used to carry out in vitro drug release studies (Electrolab, EDT-08Lx) ${ }^{[15]}$. Weighed samples, were placed in a dialysis membrane and immersed into the dissolution medium. One $\mathrm{mL}$ of 
sample was withdrawn every one hour for $12 \mathrm{~h}$ interval and replaced with equal quantity of the dissolution medium. After appropriate dilution the samples were assayed at $287 \mathrm{~nm}$. The mechanism of drug release from the nanoparticles was determined by finding Higuchi and Korsmeyer-Peppas plots ${ }^{[16-19]}$

\subsubsection{Surface morphology}

Shape and surface morphology of Levofloxacin nanoparticles were studied using scanning electron microscopy). For shape and surface morphology the nanoparticles were mounted on metal stubs and the stub was then coated with conductive gold with sputter coater attached to the instrument Jeol scanning electron microscope (SEM, JEOL-JSM-AS430, Japan) and the photographs were taken.

\subsubsection{Zeta potential determination}

The zeta potential was determined by diluting the samples with $0.1 \mathrm{mM} \mathrm{KCl}$ solution and it is adjusted to $\mathrm{pH} 7.4^{[20]}$. The surface charge of Levofloxacin nanoparticles was determined using Zetasizer Nano ZS (Malvern Instruments, Malvern, UK).

\subsubsection{Accelerated stability studies}

The selected formulations was subjected to stability by wrapping them in an aluminum foil and packed in an amber colored bottle. These nanoparticles were kept in an incubator maintained at $40 \pm 0.2{ }^{\circ} \mathrm{C}$ and $75 \pm 5 \%$ $\mathrm{RH}$ for 6 months. Changes in the appearance, drug content and in vitro dissolution studies of the stored nanoparticles were investigated.

\section{RESULTS AND DISCUSION}

\subsection{Formulation of LEV - chitosan / $\beta$-cyclodextrin nanoparticles}

LEV nanoparticles were prepared by ionic gelation method using chitosan / $\beta$-cyclodextrin. Tripolyphosphate solution were added as the cross linking in the formulation step. All the prepared formulations (six) were evaluated for particle size, drug content, drug entrapment efficiency and in vitro drug release. Depends upon the release profile the selected formulations were used for further evaluations such as surface morphology, zeta potential and stability studies.

\subsection{Particle size, drug content and drug entrapment efficiency}

The particle size and drug content of the prepared LEV nanoparticles are shown in Table 2. The particle size lies between 199 and $369 \mathrm{~nm}$ and the drug content found between $51.13 \pm 0.28$ and $71.12 \pm 0.14 \%$. The particle size of nanoparticles increased with increasing concentration of polymer matrix density and this may be due to the increased viscosity of the inner phase and decreased with increasing concentration of $\beta$ cyclodextrin. The drug entrapment efficiency differed between $59.07 \pm 1.2$ and $74.45 \pm 1.92 \%$ (Fig. 1), indicated that, this is increased with increasing concentration of chitosan and $\beta$-cyclodextrin. All the formulations showed very well drug content also.

Table 2: Particle size and drug content of $L E V$ - $\beta$-cyclodextrin

\begin{tabular}{|c|c|c|}
\hline Formulation code & Particle size $(\mathbf{n m})^{*}$ & Drug content $^{*} \boldsymbol{( \% )}$ \\
\hline G1A & 369 & $51.13 \pm 0.28$ \\
\hline G1B & 310 & $59.22 \pm 0.32$ \\
\hline G1C & 207 & $70.89 \pm 0.32$ \\
\hline G1D & 285 & $59.12 \pm 0.22$ \\
\hline G1E & 260 & $60.01 \pm 0.16$ \\
\hline G1F & 199 & $71.12 \pm 0.14$ \\
\hline
\end{tabular}

*Mean, $S D \pm n=3$

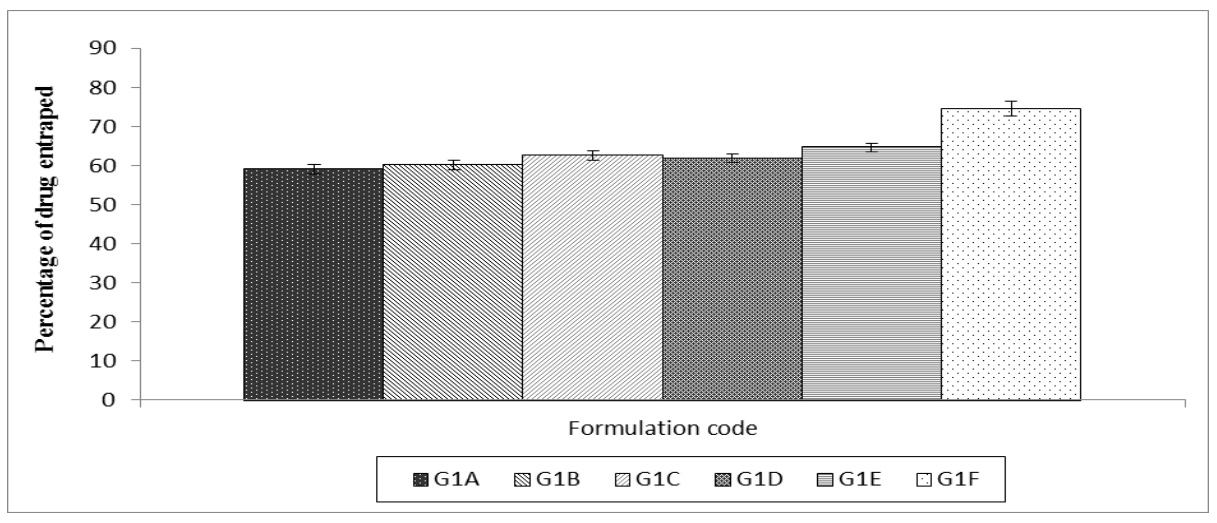

Figure 1: Drug entrapment efficiency of the formulations 


\subsection{Invitro release study}

The in vitro release of LEV from different nanoparticles is shown in Fig. 2. The maximum in vitro release was evaluated to be $91.5 \pm 2 \%$ over a period of $12 \mathrm{~h}$ for the formulation G1A. All other formulations G1B, GIC, G1D, G1E and G1F showed highest release of $>80 \%$ at the end of $12 \mathrm{~h}$. The complexation with the cyclodextrin permits a protection for sensitive drug and in vitro release profile observed for these nanoparticles was characterized by an erratic drug release in the beginning and followed by a delayed release phase. A strong reduction of cyclodextrin solubilizing efficiency towards the drug was observed, when chitosan and cyclodextrin present at the same time. Controlled drug delivery systems, release the drug through a variety of complex mechanisms, and are not yet entirely clear. This may be governed by either purely diffusion or purely erosion controlled. The results indicated that the release of the drug decreased with increasing concentration of chitosan and also, $\beta$-cyclodextrin provides an additional element for controlling the release of LEV from the formulations. Among all the formulations, G1C, G1E and G1F followed the drug release in a controlled manner and further used for surface morphology, zeta potential and stability studies.

The release data of the tested nanoparticles were analyzed on the basis of Korsmeyer-Peppas equation and Higuchi kinetics ${ }^{[16-19]}$. The $\mathrm{R}^{2}, \mathrm{k}$ and $\mathrm{n}$ values are given in Table 3. The formulations G1E and G1F were best fitted to super case II transport drug release, according to this drug release mechanism could be due to increased plasticization at the relaxing boundary. But the formulation G1C provided good fit to the Higuchi model. The drug release from these nanoparticles may be controlled by diffusion through the micropores.

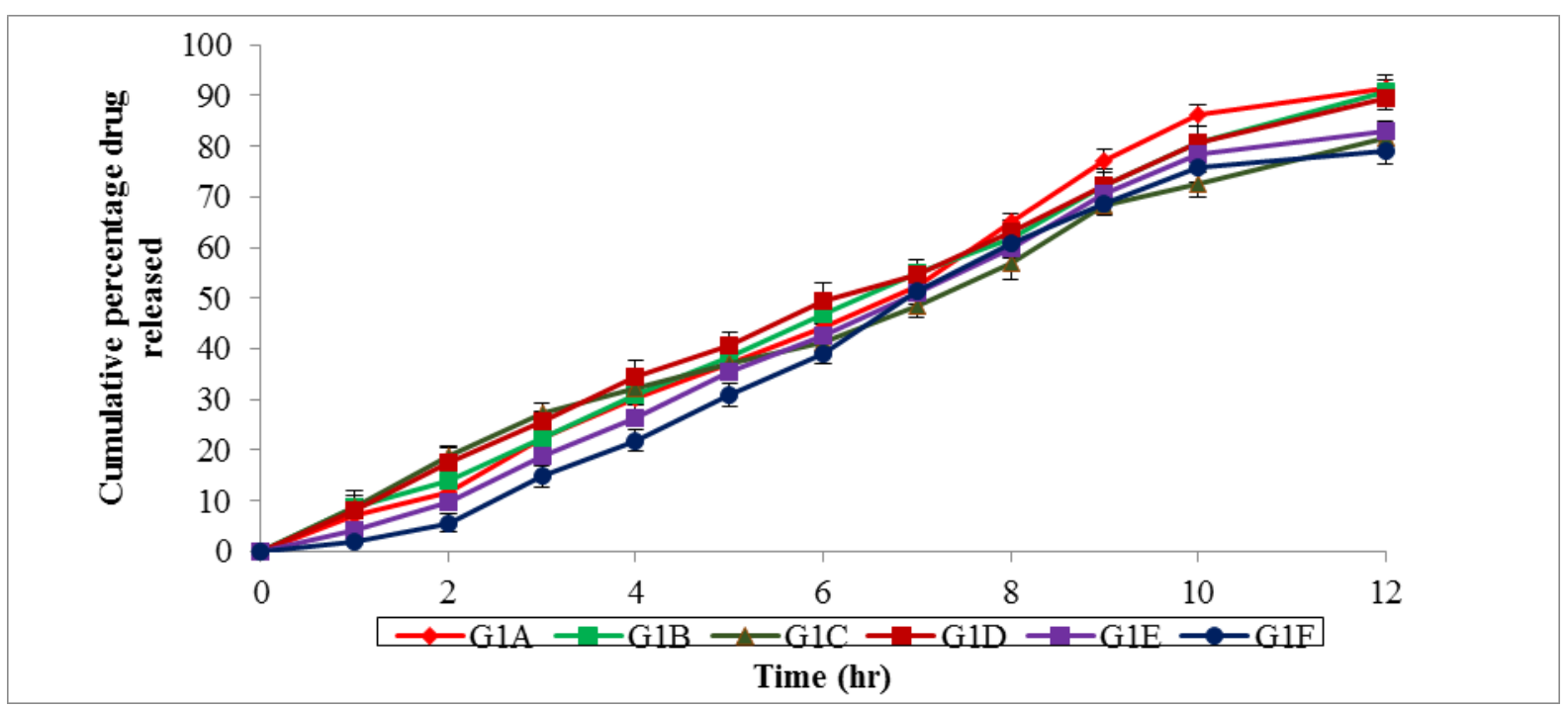

Figure 2: The in vitro drug release of the formulations

Table 3: The $\boldsymbol{R}^{2}, k$ and $n$ values of selected formulations

\begin{tabular}{|c|l|c|c|c|l|}
\hline \multirow{2}{*}{ Formulations } & \multicolumn{2}{|c|}{ Higuchi } & \multicolumn{2}{c|}{ Korsmeyer-Peppas } & \multirow{2}{*}{$\begin{array}{c}\text { Mechanism of drug } \\
\text { release }\end{array}$} \\
\cline { 2 - 5 } & $\boldsymbol{R}^{\mathbf{2}}$ & $\mathbf{k}\left(\mathbf{h}^{-\mathbf{1}}\right)$ & $\boldsymbol{R}^{\mathbf{2}}$ & $\mathbf{n}$ & \\
\hline G1C & 0.9345 & $>1$ & 0.9205 & $>1$ & Higuchi kinetics \\
\hline G1E & 0.8949 & $>1$ & 0.9050 & $>1$ & Super case II transport \\
\hline G1F & 0.870 & $>1$ & 0.8884 & $>1$ & Super case II transport \\
\hline
\end{tabular}

\subsection{Surface morphology and Zeta potential determination}

Scanning electron microscopy indicated that the prepared nanoparticles were discrete, uniform and spherical with a smooth surface. The monographs of selected formulations are shown in Fig. 3. The Zeta potential of selected formulations is shown in Table 4. The surface charge of LEV - $\beta$-cyclodextrin nanoparticles was stronger and the LEV molecules were released slowly [the amount released slowly $(<80 \%)$ within $12 \mathrm{~h}$ ] due to the electrostatic attraction between the drug and nanoparticles. This indicated that, the formation of nanoparticle depended dramatically on the concentration of free amino groups, which increase the zeta potential of the nanoparticles.

Table 4: The Zeta potential of selected formulations

\begin{tabular}{|c|c|}
\hline Formulations & $\begin{array}{c}\text { Zeta potential } \\
(\mathbf{m V}) *\end{array}$ \\
\hline G1C & $31.8 \pm 0.28$ \\
\hline G1E & $31.3 \pm 0.17$ \\
\hline G1F & $28.7 \pm 0.45$ \\
\hline \multicolumn{2}{|c}{$*$ Mean, $S D \pm n=3$}
\end{tabular}




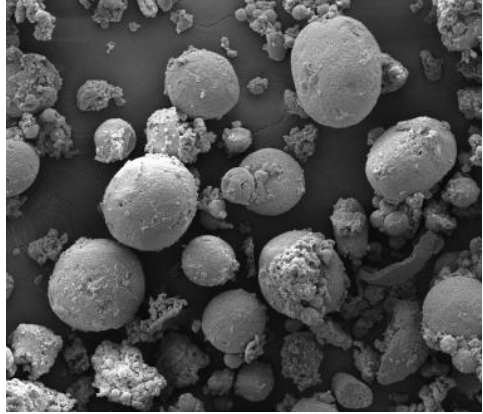

Formulation G1C

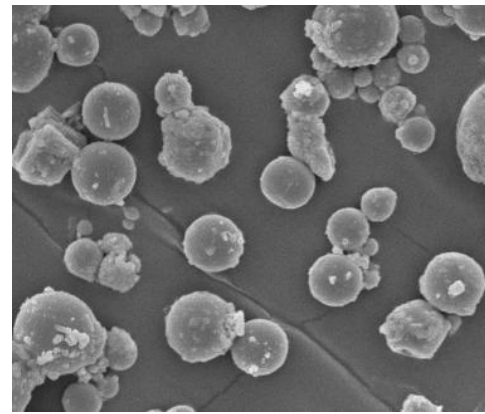

Formulation G1E

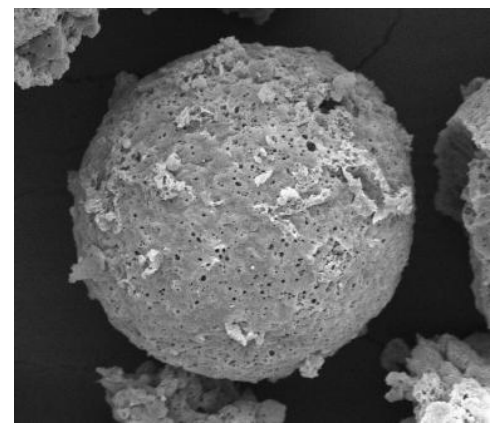

Formulation G1F

Figure 3: The surface morphology of the selected formulations

\subsection{Accelerated stability studies}

During and at the end of the stability study, the tested formulation showed almost same drug content, in vitro drug release and no color changes from that observed at the opening of the study.

\section{CONCLUSIONS}

Levofloxacin nanoparticles can be formulated using chitosan and $\beta$-cyclodextrin by ionic gelation method.

\section{REFERENCES}

1. Diren S, Zeynep, FK, Bioavailability File: Levofloxacin, J Pharm. Sci, 32, 2007, 197-208.

2. Avnesh K, Sudesh KY, Subash CY, Biodegradable polymeric nanoparticles based drug delivery system. Colloids Surf B Biointerfaces, 75, 2010, 1-18.

3. Yie WC. Concepts and System Design for Rate-controlled Drug Delivery. Novel Drug Delivery System 2nd ed. Marcel Dekker: New York.1992; 1-42

4. Arora S, Ali A, Ahuja A, Khar RK, Baboota S, Floating drug delivery systems: A review, AAPS PharmSciTech, 6, 2005, E372- E390.

5. Deshpande AA, Rhodes CT, Shah NH, Malick AW, Controlled-release drug delivery systems for prolonged gastric residence: an overview, Drug Dev Ind Pharm., 22, 1996, 631639.

6. Hwang vSJ, Park H, Park K, Gastric retentive drug-delivery systems, Crit Rev Ther Drug Carrier Syst, 15, 1998, 243-283.

7. Higuchi T, Connors K.A, Phase solubility techniques, Adv Anal Chem. Instrum, 4, 1965, 117-122.

8. Zerrouk N, Carti G, Ancillatti S, Maestrelli, F, Cirri M, Mura $\mathrm{P}$, Influence of cyclodextrins and chitosan, separately or in combination, on glyburide solubility and permeability, Eur $\mathbf{J}$ Pharm Biopharm, 62, 2006, 241-246.

9. Mukesh, CS, Smita S, Formulation and Spectroscopic Studies and Dissolution Behaviour of Levofloxacin- $\beta$ Cyclodextrins Inclusion Complex, Int J PharmTech Res, 3, 2011, 1883-1888.

10. Yaowalak B, Ampol M, Bernd WM, Chitosan drug binding by ionic interaction, Eur J Pharm Biopharm, 62, 2006, 267-74.

11. Aguerosa M, Ruiz-Gatona LC, Vauthierb K, Bouchemalb S, Espuelasa G, Ponchelb J.M, Combined hydroxypropylcyclodextrin and poly (anhydride) nanoparticles improve the oral permeability of paclitaxel, Eur J Pharm Sci, 38, 2009,
The in vitro release profile showed that maximum amount of Levofloxacin is released from the prepared nanoparticles in a controlled manner. From this, it concluded that this is a potential drug delivery for Levofloxacin and pertaining to these studies, this has to confirm in the in vivo settings as a separate investigation in future.

405-13.

12. Eric A, Robert G, Eric D, Drug loaded nanoparticlespreparation methods and drug targeting issues, Eur J Pharm Biopharm, 39, 1993, 73-91.

13. Soppimath KS, Aminabhavi TM, Kulkarni AR, Rudzinski WE, Biodegradable polymeric nanoparticles as drug delivery devices, J Control Release, 70, 2001, 1-20.

14. Yaowalak B, Ampol M, Bernd WM, Chitosan drug binding by ionic interaction, Eur J Pharm Biopharm, 62, 2006, 267-74.

15. United States Pharmacopeia/National Formulary (USP 23/NF18) (1995). 18th ed.; US Pharmacopoeial Convention: Rockwile.

16. Higuchi $\mathrm{T}$, Rate of release of medicaments from ointment bases containing drugs in suspension, J Pharm Sci, 50, 1961, 874-875.

17. Korsmeyer RW, Gurny R, Doelker E, Buri P, Peppas NA, Mechanism of potassium chloride release from compressed, hydrophilic, polymeric matrices: Effect of entrapped air, J Pharm Sci, 72, 1983, 1189-1191.

18. Puratchikody A, Prasanth VV, Sam TM, Ashok KB, Development and characterization of mucoadhesive patches of salbutamol sulphate for unidirectional buccal drug delivery, Acta Pharmaceutica, 61, 2011, 157-170.

19. Puratchikody A, Prasanth VV, Sam TM, Ashok BK., Mucoadhesive patches of salbutamol sulfate for unidirectional buccal drug delivery: Development and evaluation, Current Drug Delivery, 8, 2011, 416-425.

20. Aguerosa M, Ruiz-Gatona LC, Vauthierb K, Bouchemalb S, Espuelasa G, Ponchelb JM, Combined hydroxypropylcyclodextrin and poly (anhydride) nanoparticles improve the oral permeability of paclitaxel, Eur J Pharm Sci, 38, 2009, 40513. 